Pacific Journal of Mathematic 


\section{ON THE BANACH SPACES OF FUNCTIONS WITH BOUNDED UPPER MEANS}

\section{KA-SING LAU}

We consider the Banach space $\mathscr{M}^{p}(R)$ of functions with bounded upper means. A detailed study is made of the extremal structure of the closed unit sphere, the dual space and the representations of the bounded linear functionals on $\mathscr{A}^{p}(\boldsymbol{R})$.

1. Introduction. In his celebrated paper on generalized harmonic analysis [13], Wiener introduced the following integrated transformation

$$
s(u)=\underset{A \rightarrow \infty}{\operatorname{i} . m} \cdot \frac{1}{2 \pi}\left(\int_{-A}^{-1}+\int_{1}^{A}\right) \frac{f(x) e^{-i u x}}{-i x} d x+\frac{1}{2 \pi} \int_{-1}^{1} f(x) \frac{e^{-i u x}-1}{-i x} d x,
$$

where $f$ is a complex valued Borel measurable function on $\boldsymbol{R}$ which satisfies $\int_{-\infty}^{\infty}|f(x)|^{2} /\left(1+x^{2}\right) d x<\infty$. By using a deep Tauberian theorem, he showed that if either limit exists, then

$$
\lim _{T \rightarrow \infty} \frac{1}{2 T} \int_{-T}^{T}|f(x)|^{2} d x=\lim _{h \rightarrow 0^{+}} \frac{1}{2 h} \int_{-\infty}^{\infty}|s(u+h)-s(u-h)|^{2} d u .
$$

The formula has important applications in studying physical phenomena such as white light, noise, and turbulence where ordinary harmonic analysis is not applicable [2], [12], [13].

Unfortunately, the class $\mathscr{W}^{2}(\boldsymbol{R})$ of Borel measurable functions $f$ such that $\lim _{T \rightarrow \infty} 1 / 2 T \int_{-T}^{T}|f(x)|^{2} d x$ exists is not closed under addition. It is natural to consider a larger linear space which contains the above nonlinear space of functions. In [11], Marcinkiewicz defined the class $\mathscr{M}^{p}(\boldsymbol{R}), 1 \leqq p<\infty$, as the set of Borel measurable functions $f$ with

$$
\|f\|=\varlimsup_{T \rightarrow \infty}\left(\frac{1}{2 T} \int_{-T}^{T}|f(x)|^{p} d x\right)^{1 / p}<\infty .
$$

By identifying functions whose difference has zero norm, he proved that $\left(\mathscr{C}^{p}(\boldsymbol{R}),\|\cdot\|\right)$ is actually a Banach space. The space had been studied by many authors in the theory of almost periodic functions and generalized harmonic analysis (e.g., Besicovitch [4], Bohr and Følner [6], Bertrandias [3] and Lau and Lee [10]). In [10], it was shown that the transformation defined in (1.1) can be extended to an isomorphism from $\mathscr{M}^{2}(\boldsymbol{R})$ onto the space $\mathscr{V}^{2}(\boldsymbol{R})$ of functions with 
bounded quadratic variations (i.e., $\|s\|=\varlimsup_{h \rightarrow 0^{+}}\left(1 / 2 h \int_{-\infty}^{\infty} \mid s(u+h)-\right.$ $\left.\left.\left.s(u-h)\right|^{2} d u\right)^{1 / 2}<\infty, s \in \mathscr{V}^{2}(\boldsymbol{R})\right)$. Note that Wiener's identity (1.2) implies that transformation (1.1) is an isometry on $\mathscr{W}^{2}(\boldsymbol{R})$. The theorem revealed that $\mathscr{M}^{p}(\boldsymbol{R})$ and $\mathscr{V}^{p}(\boldsymbol{R})$ are interesting spaces and further study is desirable. In this paper, we concentrate on two topics, viz., the extremal structure of the closed unit sphere in $\mathscr{M}^{p}(\boldsymbol{R})$ and the representations of functionals on $\mathscr{L}^{p}(\boldsymbol{R})$.

In $\S 3$, we prove

THeOREM 3.8. Let $1<p<\infty$ and let $f \in \mathscr{L}^{p}(\boldsymbol{R})$ with $\|f\|=1$. Suppose there exists an increasing sequence $\left\{T_{n}\right\}$ which diverges to $\infty$, with $\left\{T_{n+1} / T_{n}\right\}$ bounded and $\lim _{n \rightarrow \infty} 1 / 2 T_{n} \int_{-T_{n}}^{T_{n}}|f(x)|^{p} d x=1$. Then $f$ is an extreme point of the closed unit sphere $S\left(\mathscr{L}^{p}(\boldsymbol{R})\right)$.

In particular, every function in $\mathscr{W}^{p}(\boldsymbol{R}), 1<p<\infty$, is an extreme point of $S\left(\mathscr{C}^{p}(\boldsymbol{R})\right)$. A partial converse of the above theorem is also given (Theorem 3.10). For $p=1$, we show that $S\left(\mathscr{C}^{1}(R)\right.$ ) does not have any extreme points (Theorem 3.11).

In order to study the dual space of $\mathscr{M}^{p}(\boldsymbol{R})$, it is convenient to make use of the following spaces:

$$
\begin{aligned}
M^{p}(\boldsymbol{R}) & =\left\{f: f \text { is Borel measurable, }\|f\|=\sup _{1 \leqq T<\infty}\left(\frac{1}{2 T} \int_{-T}^{T}|f|^{p}\right)^{1 / p}<\infty\right\}, \\
I^{p}(\boldsymbol{R}) & =\left\{f \in M^{p}(\boldsymbol{R}): \varlimsup_{T^{\prime} \rightarrow \infty} \frac{1}{2 T} \int_{-T}^{T}|f|^{p}=0\right\} .
\end{aligned}
$$

We will identify $\mathscr{M}^{p}(\boldsymbol{R})$ with the quotient space $M^{p}(\boldsymbol{R}) / I^{p}(\boldsymbol{R})$. For $1<p<\infty$, we show that $M^{p}(\boldsymbol{R})$ is the second dual of $I^{p}(\boldsymbol{R})$ and $M^{p}(\boldsymbol{R})^{*}=I^{p}(\boldsymbol{R})^{*} \oplus I^{p}(\boldsymbol{R})^{\perp}$, with $\mathscr{M}^{p}(\boldsymbol{R})^{*}$ isometric isomorphic to $I^{p}(\boldsymbol{R})^{\perp}$. By using a method of Cwikel [7] and the theorem of Bishop and Phelps [5], we will give concrete representations of functionals on $I^{p}(\boldsymbol{R})$ and $\mathscr{L}^{p}(\boldsymbol{R})$ (Theorem 4.6, Theorem 5.2).

Theorem. Suppose that $1<p<\infty$ and $1 / p+1 / q=1$.

(i) If $l \in I^{p}(\boldsymbol{R})^{*}$, then there exists $a \psi \in M^{q}(\boldsymbol{R})$ and a countably additive, positive, bounded regular Borel measure on $[1, \infty)$ such that for all $f \in I^{p}(\boldsymbol{R})$,

$$
\langle l, f\rangle=\int_{1}^{\infty}\left(\frac{1}{2 T} \int_{-T}^{T} f(x) \psi(x) d x\right) d \mu(T) .
$$

(ii) There exists a (norm) dense subset $D \subseteq \mathscr{M}^{p}(\boldsymbol{R})^{*}$ such that each $l$ in $D$ can be represented as in (1.3) with $\psi \in \mathscr{M}^{q}(\boldsymbol{R})$ and $\mu a$ 
finitely additive, positive, bounded regular Borel measure on $[1, \infty)$ concentrated at $\infty$.

We are unable to represent every functional in $\mathscr{M}^{p}(\boldsymbol{R})^{*}$. However, if we consider the subspace. $\mathscr{C l}_{r}^{p}(\boldsymbol{R})$, the $\mathscr{M}^{p}$-regular functions defined by

$$
\mathscr{C}_{r}^{p}(\boldsymbol{R})=\left\{f \in \mathscr{M}^{p}(\boldsymbol{R}): \lim _{T \rightarrow \pm \infty} \frac{1}{T} \int_{T}^{T+1}|f|^{p}=0\right\}
$$

we can show that (Theorem 5.5).

(iii) Each $l \in \mathscr{L}_{r}^{p}(\boldsymbol{R})^{*}$ can be represented as in (1.3), where $\mu$ is the same as in (ii) and $\psi$ is a Borel measurable function on $[1, \infty) \times \boldsymbol{R}$ with $\psi(T, \cdot) \in \mathscr{l}_{r}^{q}(\boldsymbol{R})$ for each $T \in[1, \infty)$.

We remark that the representations in (i), (ii), (iii) are not unique. Our paper is organized as follows: in $\S 2$, we list some relevant properties of Banach space theory and prove some elementary results for the spaces $M^{p}(\boldsymbol{R}), I^{p}(\boldsymbol{R})$ and $\mathscr{M}^{p}(\boldsymbol{R})$. In $\S 3$, we study the extreme points of $S\left(M^{p}(\boldsymbol{R})\right)$ and $S\left(\mathscr{M}^{p}(\boldsymbol{R})\right)$. In $\S 4$, we show that $I^{p}(\boldsymbol{R})^{* *}=M^{p}(\boldsymbol{R})$ and part (i) of the above theorem. These results are used in $\S 5$ to prove part (ii) and (iii) of the theorem.

The author would like to express his gratitude to Professor Masani for bringing his attention to this subject and for many helpful discussions and comments.

2. Notations and basic properties. Let $X$ be a Banach space and let $S(X)=\{f \in X:\|f\| \leqq 1\}$ be the closed unit sphere of $X$. $X^{*}$ will denote the dual space of $X$. An $l \in X^{*}$ is called a norm attaining functional if there exists an $f \in S(X)$ such that $\langle l, f\rangle=\|l\|$. The well known theorem of Bishop and Phelps [5] states that

The set of norm attaining functionals on $X$ is dense in $X^{*}$. For any closed subspace $Y$ of $X$, let $X / Y$ be the quotient space and let $Y^{\perp}$ be the annihilator of $Y$. It is elementary that $(X / Y)^{*}$ is isometrically isomorphic to $Y^{\perp}$.

A Banach space $X$ is called uniformly convex [8] if

$$
\delta(\varepsilon)=\inf \left\{1-\frac{\|f+g\|}{2}:\|f-g\| \geqq \varepsilon, f, g \in S(X)\right\}, \quad \varepsilon>0
$$

is a strictly positive function on $\boldsymbol{R}^{+}, \delta(\cdot)$ is called the modulus of convexity of $X$. If $(\Omega, \mu)$ is a measure space, it is known that $L^{p}(\Omega, \mu), 1<p<\infty$, is uniformly convex and that $\delta(\cdot)$ depends only on $\varepsilon$ and $p$ and is independent of the underlying measure space.

Let $X$ be a uniformly convex space. It follows directly from the definition that if $f, g \in S(X)$ with $\|f\|=1$ and $\|f-g\| \geqq \varepsilon$, then $\left|\left\langle l_{f}, g\right\rangle\right| \leqq 1-2 \delta(\varepsilon)$ where $l_{f}$ is a norm one functional on $X$ and 
attains its norm on $f$. We will need the following slightly stronger statement:

Lemma 2.1. Let $X$ be a uniform convex space with modulus of convexity $\delta(\cdot)$. Suppose that given $\varepsilon>0$, there exist $f, g$ in $S(X)$ and $l_{f} \in S\left(X^{*}\right)$ such that $\|f-g\| \geqq \varepsilon, 1-\varepsilon / 2 \leqq\|f\| \leqq 1$ and $l_{f}$ attains its norm at $f /\|f\|$. Then $\left|\left\langle l_{f}, g\right\rangle\right| \leqq 1-2 \delta(\varepsilon / 2)$.

Throughout, we shall assume that $f$ is a complex valued Borel measurable function on $\boldsymbol{R}$. Given a positive Borel measurable function $w(x)$, we will use $L^{p}(\boldsymbol{R}, w(x) d x)$ to be the Banach space of Borel measurable functions $f$ such that $\|f\|=\left(\int_{R}|f(x)|^{p} w(x) d x\right)^{1 / p}<\infty$. For a locally integrable function $f$, we define

$$
A(T, f)=\frac{1}{2 T} \int_{-T}^{T} f(x) d x, \quad T \geqq 1 .
$$

Let $M^{p}(\boldsymbol{R})$ and $I^{p}(\boldsymbol{R})$ be defined as in the introduction with $\|f\|=$ $\sup _{1 \leqq T<\infty} A\left(T,|f|^{p}\right)^{1 / p}$. It is known that $M^{2}(\boldsymbol{R}) \varsubsetneqq L^{2}\left(\boldsymbol{R}, d x /\left(1+x^{2}\right)\right)[14]$. We refer to [10] for the following result.

Proposition 2.2. Let $1 \leqq p<\infty$, then for any $a>0, M^{p}(\boldsymbol{R}) \varsubsetneqq$ $L^{p}\left(\boldsymbol{R}, d x /\left(1+|x|^{1+a}\right)\right)$.

Proposition 2.3. Let $1 \leqq p<\infty$, then

(i) $L^{p}(\boldsymbol{R})$ is a dense subspace in $I^{p}(\boldsymbol{R})$ and $I^{p}(\boldsymbol{R})$ is separable;

(ii) $I^{p}(\boldsymbol{R})$ contains a subspace isomorphic to $c_{0}$.

Proof. We omit the simple proof of (i). To show that $I^{p}(\boldsymbol{R})$ contains a $c_{0}$, we proceed as follows: let $n_{1}=1, f_{1}=4^{1 / p} \chi_{[1,2]}$ and choose for $k>1, n_{k}$ and $f_{k}$ such that $1<n_{1}+1<n_{2}<\cdots<n_{k-1}+$ $1<n_{k}, f_{k}=\left(2\left(n_{k}+1\right)\right)^{1 / p} \chi_{\left[n_{k}, n_{k+1}\right]}$ and

$$
\frac{1}{2 n_{k}} \int_{0}^{n_{k-1}+1} \sum_{j=1}^{k-1} f_{j}(x)^{p} d x<\frac{1}{2}
$$

Clearly, $\left\|f_{k}\right\|=1$. We claim that the subspace generated by $\left\{f_{k}\right\}$ is isomorphic to $c_{0}$. If $\left\{c_{k}\right\}$ is a sequence in $c_{0}$ such that $\sup _{k}\left|c_{k}\right|=1$, then for any $T$, we can find a $k$ such that $n_{k} \leqq T<n_{k+1}$. Thus by our construction of $\left\{f_{k}\right\}$,

$$
A\left(T,\left|\sum_{k=1}^{\infty} c_{k} f_{k}\right|^{p}\right) \leqq \frac{1}{2 n_{k}} \int_{0}^{n_{k}+1} \sum_{j=1}^{k}\left|c_{j}\right|^{p} f_{j}^{p} \leqq \frac{n_{k}+1}{n_{k}}\left|c_{k}\right|^{p}+\frac{1}{2}<3 .
$$

Hence $1 \leqq\left\|\sum_{k=1}^{\infty} c_{k} f_{k}\right\| \leqq 3^{1 / p}$ for any $\left\{c_{k}\right\}$ in $c_{0}$ with $\sup _{k}\left|c_{k}\right|=1$ and the claim is proved. 
Let $\mathscr{C}^{p}(\boldsymbol{R}), 1 \leqq p<\infty$ be the set of measurable functions on $\boldsymbol{R}$ such that $\|f\|=\varlimsup_{\lim _{T \rightarrow \infty}} A\left(T,|f|^{p}\right)^{1 / p}<\infty$. By identifying functions whose difference has zero norm, $\mathscr{C}^{p}(\boldsymbol{R})$ is a Banach space [11]. Let $\mathscr{U}^{p}(\boldsymbol{R})$ be the set of $f \in \mathscr{C}^{p}(\boldsymbol{R})$ such that $\lim _{T \rightarrow \infty} A\left(T,|f|^{p}\right)$ exists. Note that $\mathscr{W}^{p}(\boldsymbol{R})$ is a nonlinear subspace. The following identification of $\mathscr{C}^{p}(\boldsymbol{R})$ will be very useful for us. The proof is in $[10]$.

Proposition 2.4. $\mathscr{M}^{p}(\boldsymbol{R})$ is isometric isomorphic to $M^{p}(\boldsymbol{R}) / I^{p}(\boldsymbol{R})$ under the natural identification.

Proposition 2.5. $\mathscr{M}^{p}(\boldsymbol{R})$ contains a subspace isomorphic to $l^{\infty}$. Consequently, $\mathscr{L}^{p}(\boldsymbol{R})$ is nonseparable and nonreflexive.

Proof. Let $a_{1}=0, b_{1}=1$ and $a_{n}=2^{n} b_{n-1}, b_{n}=2^{n} a_{n}$. Then

$$
\frac{1}{a_{n}} \int_{-a_{n}}^{a_{n}} \chi_{\left[a_{n-1}, b_{n-1}\right]}<\frac{1}{2^{n}} \quad \text { and } \quad \frac{1}{b_{n}} \int_{-b_{n}}^{b_{n}} \chi_{\left[a_{n}, b_{n}\right]}=1-\frac{1}{2^{n}} \text {. }
$$

Let $\left\{\mathscr{T}_{n}\right\}_{n \in N}$ be a partition of the set of natural number $N$ such that each $\mathscr{I}_{n}$ is an infinite set. Let $f_{n}=2 \sum_{k \in r_{n}} \chi_{\left[a_{k}, b_{k}\right]}$, note that $\varlimsup_{T \rightarrow \infty} A\left(T,\left|f_{n}\right|^{p}\right)=1$ for each $n$. If $\left\{c_{n}\right\}$ is a sequence such that $\sup _{n}\left|c_{n}\right|=1$, then it is clear that $1 \leqq\left\|\sum_{n=1}^{\infty} c_{n} f_{n}\right\|$. For each $T$, there exists a $k$ such that $a_{k} \leqq T<a_{k+1}$. Hence

$$
\begin{aligned}
A\left(T,\left|\sum_{n=1}^{\infty} c_{n} f_{n}\right|^{p}\right) & \leqq A\left(T,\left|\sum_{j=1}^{k} \chi_{\left[a_{j}, b_{j}\right]}\right|^{p}\right) \\
& \leqq \frac{1}{2 T} \int_{-T}^{T} \chi_{\left[a_{k}, b_{k}\right]}+\frac{1}{2 a_{k}} \int_{-a_{k}}^{a_{k}} \sum_{j=1}^{k-1} \chi_{\left[a_{j}, b_{j}\right]} \\
& \leqq 1+\frac{1}{2} \sum_{j=1}^{k-1} \frac{1}{2^{j}} \\
& \leqq 2 .
\end{aligned}
$$

Thus $1 \leqq\left\|\sum_{n=1}^{\infty} c_{n} f_{n}\right\| \leqq 2^{1 / p}$ and this induces an isomorphism from $l^{\infty}$ onto the subspace generated by $\left\{f_{n}\right\}$ in $\mathscr{M}^{p}(\boldsymbol{R})$.

Let $B^{p} A P$ be the class of (Besicovitch) almost periodic functions, the $\mathscr{C}^{p}$-closure of the set of trigonometric polynomials $\sum_{k=1}^{n} a_{k l} e^{i t_{l_{l}}(\cdot)}$, $t_{i b} \in \boldsymbol{R}$. It is known that $B^{p} A P$ is a closed subspace of $\mathscr{W}^{p p}(\boldsymbol{R})([6$, p. 45]). For the case $p=2$, we can define an inner product by

$$
(f, g)=\lim _{T \rightarrow \infty} A(T, f \bar{g}), \quad f, g \in B^{2} A P .
$$

This inner product induces a norm on $B^{2} A P$ which coincides with the $\mathscr{C}^{2}$-norm. It follows that $\mathscr{C}^{2}(\boldsymbol{R})$ contains a nonseparable Hilbert space (since $f_{t}(\cdot)=e^{i t(\cdot)} \in B^{2} A P$ for all $t \in \boldsymbol{R}$ ). 
Proposition 2.6. For $1<p<\infty, \mathscr{C}^{p}(\boldsymbol{R})$ contains a nonseparable reflexive Banach space.

Proof. It follows from the definition of $B^{p} A P$ and the Hölder inequality that for $f \in B^{p} A P, g \in B^{q} A P, 1 / p+1 / q=1, f g \in B^{1} A P$, hence $\lim _{T \rightarrow \infty} A(T, f g)$ exists. By defining $\langle g, f\rangle=\lim _{T \rightarrow \infty} A(T, f g)$, we can show that $\left(B^{p} A P\right)^{*}=B^{q} A P$ and $\left(B^{q} A P\right)^{*}=B^{p} A P$. Hence, $B^{p} A P$ is reflexive. Observe that it is also nonseparable. This proves the proposition.

3. Extreme points. Let $K$ be a convex subset in a linear space $X . f \in K$ is called an extreme point of $K$ if for any $g, h \in K$ such that $f=\lambda g+(1-\lambda) h, 0<\lambda<1$, then $f=g=h$. The definition is equivalent to the statement: $\forall g \in X, f \pm g \in K$ implies that $g=0$.

Lemma 3.1. Let $f \in M^{p}(\boldsymbol{R}), 1 \leqq p<\infty$. Then $A\left(T,|f|^{p}\right)=1$ for all $T \geqq 1$ if and only if $|f(x)|^{p}+|f(-x)|^{p}=2$ for almost all $x \geqq 1$.

Proof. The sufficiency is obvious. To prove the necessity, observe that $A\left(T,|f|^{p}\right)=1 / 2 T \int_{-T}^{T}|f|^{p}$ is absolutely continuous on $T$. Differentiation yields that

$$
-\frac{1}{2 T^{2}} \int_{-T}^{T}|f|^{p}+\frac{1}{2 T}\left(|f(T)|^{p}+|f(-T)|^{p}\right)=0 \quad \text { a.a. } \quad T \geqq 1
$$

and this implies $|f(x)|^{p}+|f(-x)|^{p}=2$ for almost all $x \geqq 1$.

THeOREM 3.2. Let $1<p<\infty$ and let $f \in S\left(M^{p}(\boldsymbol{R})\right)$.

(i) Suppose there exists $a c>0$ and a sequence $\left\{T_{n}\right\}$ diverging to $\infty$ with $A\left(T_{n},|f|^{p}\right)^{1 / p}>1-\delta\left(\left(c / T_{n}\right)^{1 / p}\right)$, where $\delta(\cdot)$ is the modulus of convexity of $L^{p}$. Then $f$ is an extreme point of $S\left(M^{p}(\boldsymbol{R})\right)$. Conversely,

(ii) Suppose $f$ is an extreme point of $S\left(M^{p}(\boldsymbol{R})\right)$. Then for any $c>0$, there exists a sequence $\left\{T_{n}\right\}$ diverging to $\infty$ such that $A\left(T_{n},|f|^{p}\right)^{1 / p}>1-\left(c / T_{n}\right)^{1 / p}$.

REMARK. Geometrically, condition (i) says that if there exists a sequence $\left\{T_{n}\right\}$ such that $A\left(T_{n},|f|^{p}\right) \rightarrow 1$ sufficiently fast, then $f$ is an extreme point of $S\left(M^{p}(\boldsymbol{R})\right)$.

Proof. (i ) Suppose there exists a $g \in M^{p}(\boldsymbol{R})$ such that $\|f \pm g\| \leqq 1$ and $g \neq 0$ on $\left[-T_{0}, T_{0}\right]$ for some $T_{0}>0$. Let $c=\int_{-T_{0}}^{T_{0}}|g|^{p}$. The uniform convexity of $L^{p}([-T, T], d x / 2 T), T>T_{0}$ and the fact that 
$1 / 2 T \int_{-T}^{T}|(f+g)-(f-g)|^{p} \geqq c / T$ yield $A\left(T,|f|^{p}\right)^{1 / p} \leqq 1-\delta\left((c / T)^{1 / p}\right)$. This is a contradiction.

Suppose statement (ii) is false. Then there exists a $c>0$ such that for $T>T_{0}, A\left(T,|f|^{p}\right)^{1 / p}+(c / T)^{1 / p} \leqq 1$. If $g=(2 c)^{1 / p} \chi_{\left[T_{0}, T_{0}+1\right]}$, then

$$
A\left(T,|f \pm g|^{p}\right)^{1 / p} \leqq A\left(T,|f|^{p}\right)^{1 / p}+(c / T)^{1 / p} \leqq 1
$$

This implies $f$ is not an extreme point of $S\left(M^{p}(R)\right)$.

COROLlaRy 3.3. Let $1<p<\infty$ and let $f \in M^{p}(\boldsymbol{R})$ such that $|f(x)|^{p}+|f(-x)|^{p}=2$ a.e. Then $f$ is an extreme point of $S\left(M^{p}(\boldsymbol{R})\right)$.

Proof. The result follows directly from Lemma 3.1 and Theorem 3.2 .

Clarkson proved that on $L^{p}$, the modulus of convexity satisfies

$$
\delta(\varepsilon)= \begin{cases}1-\left(1-\left(\frac{\varepsilon}{2}\right)^{p}\right)^{1 / p}, & 2 \leqq p<\infty \\ \frac{p-1}{8} \varepsilon^{2}+\cdots \geqq \frac{p-1}{8} \varepsilon^{2}, & 1<p<2,\end{cases}
$$

[8, p. 149]. By considering $\varepsilon=(2 c / T)^{1 / p}$ for some $c>0$, the following results are obtained:

Corollary 3.4. Let $2 \leqq p<\infty$ and let $f \in S\left(M^{p}(\boldsymbol{R})\right)$. Suppose there exists a $c>0$ and a sequence $\left\{T_{n}\right\}$ diverging to $\infty$ such that $A\left(T_{n},|f|^{p}\right)>1-\left(c / T_{n}\right)$. Then $f$ is an extreme point of $S\left(M^{p}(R)\right)$.

Corollary 3.5. Let $1<p<2$ and let $f \in S\left(M^{p}(\boldsymbol{R})\right)$. Then the same conclusion holds if we replace the above inequality by $A\left(T_{n},|f|^{p}\right)^{1 / p}>1-\left(c / T_{n}\right)^{2 / p}$.

For the case $p=1$, we have

THEOREM 3.6. $S\left(M^{1}(\boldsymbol{R})\right)$ contains no extreme point.

Proof. Let $f \in S\left(M^{1}(\boldsymbol{R})\right)$ and $\|f\|=1$. If $\int_{-1}^{1}|f|=a>0$, by the fact that $L^{1}$ contains no extreme point, we can find a nonzero $g$ which vanishes outside $[-1,1]$ and $\int_{-1}^{1}|f \pm g|=a$. Hence

$$
A(T,|f \pm g|) \leqq 1 \quad \text { for all } \quad T \geqq 1
$$


and $f$ is not an extreme point of $S\left(M^{1}(R)\right)$. If $\int_{-1}^{1}|f|=0$, choose $T_{0}$ such that for $1 \leqq T \leqq T_{0}$,

$$
0<\frac{1}{2 T} \int_{-T}^{T}|f| \leqq \frac{1}{2} .
$$

By the same argument as about, we can find a $g$ such that $0<$ $\int_{-T_{0}}^{T_{0}}|g| \leqq 1 / 2, g$ vanishes outside $\left[-T_{0}, T_{0}\right]$ and

$$
\int_{-T_{0}}^{T_{0}}|f \pm g|=\int_{-T_{0}}^{T_{0}}|f|
$$

Again we have $A(T,|f \pm g|) \leqq 1$ for all $T \geqq 1$ and $f$ is not an extreme point of $S\left(M^{1}(\boldsymbol{R})\right)$.

The argument in Theorem 3.2 and Theorem 3.6 also implies the following result.

Proposition 3.7. For $1 \leqq p<\infty, S\left(I^{p}(\boldsymbol{R})\right)$ does not contain an extreme point.

In the rest of this section, we will consider the extreme points of $S\left(\mathscr{M}^{p}(\boldsymbol{R})\right)$.

Theorem 3.8. Let $1<p<\infty$ and let $f \in S\left(\mathscr{M}^{p}(\boldsymbol{R})\right)$. Suppose there exists a sequence $\left\{T_{n}\right\}$ diverging to $\infty$, such that $\left\{T_{n+1} / T_{n}\right\}$ is bounded and $\lim _{n \rightarrow \infty} A\left(T_{n},|f|^{p}\right)=1$. Then $f$ is an extreme point of $S\left(\mathscr{C l}^{p}(\boldsymbol{R})\right)$.

Proof. Suppose $g$ in $\mathscr{C}^{p}(\boldsymbol{R})$ is such that $\varlimsup_{\lim _{T \rightarrow \infty}} A\left(T,|f \pm g|^{p}\right) \leqq 1$. We claim that $\lim _{n \rightarrow \infty} A\left(T_{n},|g|^{p}\right)=0$ where $\left\{T_{n}\right\}$ is the sequence in the hypothesis. For otherwise, by passing to subsequence if necessary, we may assume that $A\left(T_{n},|g|^{p}\right) \geqq \varepsilon$ for some $\varepsilon>0$. For each $n$, consider $f, f \pm g$ as elements of $L^{p}\left(\left[-T_{n}, T_{n}\right], d x / 2 T_{n}\right)$. The uniform convexity of the $L^{p}$-norm implies that there exists a $\delta(\varepsilon)>0$ such that $A\left(T_{n},|f|^{p}\right)<1-\delta$. This contradicts the hypothesis that $\lim _{n \rightarrow \infty} A\left(T_{n},|f|^{p}\right)=1$ and the claim is proved. If $T>0$, then $T_{n} \leqq$ $T<T_{n+1}$ for some $n$. Hence

$$
\begin{aligned}
A\left(T,|g|^{p}\right) & \leqq \frac{1}{2 T} \int_{-T_{n+1}}^{T_{n+1}}|g|^{p} \leqq \frac{T_{n+1}}{T_{n}} \cdot \frac{1}{2 T_{n+1}} \int_{-T_{n+1}}^{T_{n+1}}|g|^{p} \\
& =\frac{T_{n+1}}{T_{n}} A\left(T_{n+1},|g|^{p}\right) .
\end{aligned}
$$

The boundedness of $\left\{T_{n+1} / T_{n}\right\}$ implies that the last term tends to 0 
as $T \rightarrow \infty$. Therefore $\|g\|=0$ and $f$ is an extreme point of $S\left(\mathscr{L}^{p}(\boldsymbol{R})\right)$.

COROLlary 3.9. Let $1<p<\infty$ and let $f \in \mathscr{W}(\boldsymbol{R})$ with $\|f\|=1$. Then $f$ is an extreme point of $S\left(\mathscr{l l}^{p}(\boldsymbol{R})\right)$.

It is easy to construct an extreme point of $S\left(\mathscr{C}^{p}(\boldsymbol{R})\right)$ which is not in $\mathscr{W}^{p}(\boldsymbol{R})$. For example, let $0<a<b<1$ and let $\left\{\alpha_{n}\right\}$ be a sequence such that $\alpha_{1}=1, \alpha_{n} b+1<\alpha_{n+1} a$ and $\lim _{n \rightarrow \infty} \alpha_{n}=\infty$. Let

$$
f(x)= \begin{cases}1 & |x|<a \\ 0 & \alpha_{n} a \leqq|x|<\alpha_{n} b \\ ((b+1)-a)^{1 / p}, & \alpha_{n} b<|x|<\alpha_{n} b+1 \\ 1 & \alpha_{n} b+1<|x|<\alpha_{n+1} a .\end{cases}
$$

Then we have $A\left(T,|f|^{p}\right) \leqq 1$ for all $T>0$, and $A\left(T,|f|^{p}\right)=1$ for $T \in \boldsymbol{R} \backslash \bigcup_{n=1}^{\infty}\left(\alpha_{n} a, \alpha_{n} b+1\right)$ and $A\left(\alpha_{n} b,|f|^{p}\right)=a / b<1$. This shows that $f \in S\left(\mathscr{H}^{p}(\boldsymbol{R})\right) \backslash \mathscr{W}^{p}(\boldsymbol{R})$ and $f$ satisfies the condition in Theorem 3.8, hence it is an extreme point.

In the following, we will give a partial converse to Theorem 3.8 .

Theorem 3.10. Let $1<p<\infty$ and let $f \in S\left(\mathscr{L}^{p}(\boldsymbol{R})\right)$. Suppose there exists an $\alpha$ in $(0,1)$ such that

(i ) $\left\{T>0: A\left(T,|f|^{p}\right) \geqq 1-\alpha\right\}=\bigcup_{n=1}^{\infty}\left[a_{n}, b_{n}\right]$ where $b_{n}<a_{n+1}$ and $\lim _{n \rightarrow \infty} a_{n}=\lim _{n \rightarrow \infty} b_{n}=\infty$.

(ii) $\left\{a_{n+1} / b_{n}\right\}$ is an unbounded sequence.

Then $f$ is not an extreme point of $S\left(\mathscr{C l}^{p}(\boldsymbol{R})\right)$.

REMARK. The hypotheses of the theorem essentially mean that if $A\left(T,|f|^{p}\right)$ stays below $(1-\alpha)$ infinitely often and long enough, then $f$ is not an extreme point of $S\left(\mathscr{C}^{p}(\boldsymbol{R})\right)$. A simple example of such $f$ is provided in the proof of Proposition 2.5. We also note that conditions (i) and (ii) are equivalent to: there exists an $\alpha$ in $(0,1)$ such that no sequence $\left\{T_{n}\right\}$ will satisfy $\lim _{n \rightarrow \infty} T_{n}=\infty,\left\{T_{n+1} / T_{n}\right\}$ is bounded and $\lim _{n \rightarrow \infty} A\left(T_{n},|f|^{p}\right)>1-\alpha$. (Compare this with Theorem 3.8.)

Proof. Without loss of generality we assume that $\|f\|=1$. Also, by passing to a subsequence, we assume that for each $n$, there exists a $T \in\left[a_{n}, b_{n}\right]$ such that $A\left(T,|f|^{p}\right) \geqq 1-\alpha / 2$ and that $\lim _{n \rightarrow \infty} a_{n+1} / b_{n}=\infty$. If $c_{n}=\sup \left\{T \in\left[a_{n}, b_{n}\right]: A\left(T,|f|^{p}\right)=1-\alpha / 2\right\}$, then for all $T$ in $\left[c_{n}, b_{n}\right], A\left(T,|f|^{p}\right) \leqq 1-\alpha / 2$. Define $B=\bigcup_{n=1}^{\infty} B_{n}$ where 
$B_{n}=\left[-b_{n},-c_{n}\right] \cup\left[c_{n}, b_{n}\right]$. We will consider the following two cases:

(i) Suppose $\lim _{n \rightarrow \infty} A\left(b_{n},\left|f \chi_{B_{n}}\right|^{p}\right)=0$. This implies that there is a subsequence $\left\{b_{n_{k}}\right\}$ such that $\lim _{k \rightarrow \infty} A\left(b_{n_{k}},\left|f \chi_{B_{n_{k}}}\right|^{p}\right)=0$ and yet another subsequence $\left\{b_{n_{k^{\prime}}}\right\}$ of $\left\{b_{n_{k}}\right\}$ such that $\lim _{k^{\prime} \rightarrow \infty} A\left(b_{n_{k^{\prime}}},\left|f \chi_{U_{j^{\prime} B_{n^{\prime}}}}\right|^{p}=0\right.$. In order to dispense with cumbersome notation, we assume that $\left\{n_{k^{\prime}}\right\}=\{n\}$ and by adjusting a zero function in $\mathscr{C}^{p}(\boldsymbol{R})$, we assume that $f \chi_{U_{n} B_{n}}=0$. Hence $f \chi_{B_{n}}=0$ for each $n$ and

$$
A\left(c_{n},|f|^{p}\right)=1-\frac{\alpha}{2} \quad \text { and } \quad A\left(b_{n},|f|^{p}\right)=\frac{1}{2 b_{n}} \int_{-c_{n}}^{c_{n}}|f|^{p}=1-\alpha .
$$

Subtraction yields that

$$
\left(\frac{b_{n}-c_{n}}{b_{n}}\right)=\frac{\alpha}{2-\alpha} .
$$

Let $0<a^{p}<1 / 2(2-\alpha)$, we claim that $A\left(T,\left|f \pm a \chi_{B_{n}}\right|^{p}\right) \leqq 1+$ $1 / 2\left(b_{n} / a_{n+1}\right)$ for all $T>0$. This is clear if $0<T \leqq c_{n}$. For $c_{n}<T \leqq b_{n}$, we have

$$
\begin{aligned}
A\left(T,\left|f \pm a \chi_{B_{n}}\right|^{p}\right) & =A\left(T,|f|^{p}\right)+2 a^{p} \cdot \frac{T-c_{n}}{2 T} \\
& \leqq\left(1-\frac{\alpha}{2}\right)+a^{p} \cdot \frac{b_{n}-c_{n}}{b_{n}} \\
& =\left(1-\frac{\alpha}{2}\right)+\frac{a^{p} \alpha}{2-\alpha} \\
& \leqq 1 .
\end{aligned}
$$

A similar proof shows that $A\left(T,\left|f \pm a \chi_{B_{n}}\right|^{p}\right) \leqq 1$ for $b_{n} \leqq T<a_{n+1}$. If $a_{n+1} \leqq T$, then

$$
A\left(T,\left|f \pm a \chi_{B_{n}}\right|^{p}\right) \leqq 1+2 a^{p} \cdot \frac{b_{n}}{2 a_{n+1}} \leqq 1+\frac{b_{n}}{a_{n+1}}
$$

and the claim is proved.

Choose a subsequence $\left\{n_{k}\right\}$ of $\{n\}$ with $n_{1}=1$ and $n_{k+1}$ such that for $T>n_{k+1}$,

$$
A\left(T,\left|\sum_{j=1}^{k} a \chi_{B_{n_{i}}}\right|^{p}\right) \leqq \frac{1}{k+1} .
$$

Let $g=\sum_{k=1}^{\infty} a \chi_{B_{n_{k}}} . \quad$ Then $g \in \mathscr{A}^{p}(\boldsymbol{R})$ and $\overline{\lim }_{T \rightarrow \infty} A\left(T,|g|^{p}\right) \geqq$ $a^{p} \alpha /(2-\alpha)>0$. Given $T>0$, then $n_{k}<T<n_{k+1}$ for some $k$ and

$$
\begin{aligned}
A\left(T,|f \pm g|^{p}\right)^{1 / p} & \leqq A\left(T,\left|f \pm a \chi_{B_{n_{k}}}\right|^{p}\right)^{1 / p}+A\left(T,\left|\sum_{j=1}^{k-1} a \chi_{B_{n_{j}}}\right|^{p}\right)^{1 / p} \\
& \leqq\left(1+\frac{b_{n_{k}}}{a_{n_{k}}+1}\right)^{1 / p}+\left(\frac{1}{k}\right)^{1 / p} .
\end{aligned}
$$


This implies that $\varlimsup_{\lim } A\left(T,|f \pm g|^{p}\right)=1$ with $g \neq 0$. Hence $f$ is not an extreme point of $S\left(\mathscr{K}^{p}(\boldsymbol{R})\right)$.

(ii) Suppose $\lim _{n \rightarrow \infty} A\left(b_{n},\left|f \chi_{B_{n}}\right|^{p}\right)>0$. Let $0<a<1$ be such that $0<\left|(1 \pm a)^{p}-1\right|<\alpha / 2$. For each $n$, we claim that

$$
A\left(T,\left|f \pm a f \chi_{B_{n}}\right|^{p}\right) \leqq 1+\frac{\alpha}{2} \frac{b_{n}}{a_{n+1}} .
$$

Indeed, if $c_{n} \leqq T \leqq a_{n+1}$, we have

$$
\begin{aligned}
A\left(T,\left|f \pm a f \chi_{B_{n}}\right|^{p}\right) & \leqq A\left(T,|f|^{p}\right)+\left|(1 \pm a)^{p}-1\right| \frac{1}{2 T} \int_{-T}^{T}\left|f \chi_{B_{n}}\right|^{p} \\
& \leqq\left(1-\frac{\alpha}{2}\right)+\left|(1 \pm a)^{p}-1\right| \leqq 1
\end{aligned}
$$

If $a_{n+1} \leqq T$, then

$$
\begin{aligned}
A\left(T,\left|f \pm a f \chi_{B_{n}}\right|^{p}\right) & \leqq 1+\left|(1 \pm a)^{p}-1\right| \cdot \frac{b_{n}}{a_{n+1}} \cdot \frac{1}{2 b_{n}} \int_{-b_{n}}^{b_{n}}|f|^{p} \\
& \leqq 1+\frac{\alpha}{2} \frac{b_{n}}{a_{n+1}} .
\end{aligned}
$$

This proves the claim. The same argument as in the last paragraph of part (i) enables us to derive a contradiction by choosing a $g \in$ $\mathscr{C}^{p}(\boldsymbol{R})$ with $\|g\| \neq 0$ and $\|f \pm g\| \leqq 1$.

THEOREM 3.11. The set $S\left(\mathscr{C l}^{1}(\boldsymbol{R})\right)$ contains no extreme points.

Proof. Let $f \in S\left(\mathscr{M}^{1}(\boldsymbol{R})\right)$ with $\|f\|=1, B_{1}=\left[-T_{1}, T_{1}\right]$ where $\int_{B_{1}}|f|=1$ and let $B_{n+1}=\left[-T_{n+1}, T_{n+1}\right] \backslash\left[-T_{n}, T_{n}\right]$ where $\int_{B_{n+1}}|f|=1$. It is easy to show that $T_{n} \rightarrow \infty$. Let $g=1 / 2\left(\chi_{\cup B_{2 n+1}}-\chi_{\cup B_{2 n}}\right) f$. Then $\|g\|=1 / 2$. For any $T, T_{n} \leqq T<T_{n+1}$ for some $n$, it follows from the construction that

$$
|A(T,|f \pm g|)-A(T,|f|)| \leqq \frac{1}{2 T} \int_{B_{n}}|f|=\frac{1}{2 T} .
$$

Hence $\|f \pm g\| \leqq 1$ and $f$ is not an extreme point of $S\left(\mathscr{M}^{1}(\boldsymbol{R})\right)$.

4. $I^{p}(\boldsymbol{R})^{*}$ and $M^{p}(\boldsymbol{R})^{*}$. Let $K$ be a topological space and let $C(K)$ denote the set of bounded continuous functions on $K$. Let rca $(K)(\mathrm{rba}(K))$ denote the set of countably (finitely, respectively) additive, bounded regular Borel measures on $K$. From the Hölder inequality we obtain this result.

Proposition 4.1. Let $1<p, q<\infty$ and $1 / p+1 / q=1$. Let 
$\psi \in M^{q}(\boldsymbol{R})$ and $\mu \in \mathrm{rca}[1, \infty)$. If $l: I^{p}(\boldsymbol{R}) \rightarrow \boldsymbol{C}$ is defined by

$$
\langle l, f\rangle=\int_{1}^{\infty} A(T, f \psi) d u(T), \quad f \in I^{p}(\boldsymbol{R}),
$$

then $l \in I^{p}(\boldsymbol{R})^{*}$ and

$$
\frac{1}{\|\psi\|^{q-1}} \int_{1}^{\infty} A\left(T,|\psi|^{q}\right) d \mu \leqq\|l\| \leqq \int_{1}^{\infty} A\left(T,|\psi|^{q}\right)^{1 / q} d \mu
$$

In this section, we will consider the converse of Proposition 4.1, i.e., can each $l \in I^{p}(\boldsymbol{R})^{*}$ be represented by (4.1)? For $1<p<\infty$, let $K_{p}=[1, \infty) \times S\left(M^{q}(\boldsymbol{R})\right), 1 / p+1 / q=1$, be equipped with the product topology.

Lemma 4.2. Let $1<p<\infty$. For each $f \in M^{p}(\boldsymbol{R})$, define $\tilde{f}$ as

$$
\widetilde{f}(T, \phi)=A(T, f \phi), \quad(T, \phi) \in K_{p} .
$$

Then $\sim$ is an isometric isomorphism from $M^{p}(\boldsymbol{R})$ into $C\left(K_{p}\right)$.

Proof. The Hölder inequality implies that

$$
|\tilde{f}(T, \phi)|=|A(T, f \phi)| \leqq A\left(T,|f|^{p}\right)^{1 / p} \cdot A\left(T,|\phi|^{q}\right)^{1 / q} \leqq A\left(T,|f|^{p}\right)^{1 / p} .
$$

Hence $\|\tilde{f}\|_{C\left(K_{p}\right)} \leqq\|f\|_{M p(R)}$. On the other hand, by taking $\phi_{0}=$ $(|f| /|| f||)^{p-1} \operatorname{sgn} \widetilde{f}$, we have

$$
\|\tilde{f}\|_{C\left(K_{p}\right)} \geqq \sup _{1 \leqq T} A\left(T, f \dot{\phi}_{0}\right)=\sup _{1 \leqq T} A\left(T,|f|^{p}\right)^{1 / p}=\|f\|_{M^{p}(\boldsymbol{R})} .
$$

Henceforth we will not distinguish $f$ and $\tilde{f}, f \in M^{p}(\boldsymbol{R})$. For a normal topological space $K$, we will use $\beta(K)$ to denote its StoneCěch compactification. It is known that every bounded continuous function on $K$ has a unique norm preserving extension to $\beta(K)$. Hence one can identify $C(K)$ and $C(\beta(K))$. This identification induces an isometric isomorphism from $\mathrm{rba}(K)$ onto $\mathrm{rca}(\beta(K))$. For each $\mu \in \mathrm{rca}(\beta(K))$, if we let $\nu(E)=\mu(E)$ where $E$ is a Borel subset in $K$, then $\nu \in \operatorname{rba}(K)$ and $\int_{K} f d \nu=\int_{\beta(K)} \tilde{f} d u$ for all $f \in C(K)$, where $\tilde{f}$ is the extension of $f$ on $\beta(K)$.

LEMMA 4.3. Let $1<p<\infty$ and let $l$ be a norm attaining functional in $M^{p}(\boldsymbol{R})^{*}$. Then there exists $a \psi \in S\left(M^{q}(\boldsymbol{R})\right)$ and a positive $\mu \in \operatorname{rba}[1, \infty)$ such that $\|\mu\|=\|l\|$ and

$$
\langle l, f\rangle=\int_{1}^{\infty} A(T, f \psi) d \mu(T) \quad \forall f \in M^{p}(\boldsymbol{R}) .
$$


Proof. We will identify $M^{p}(\boldsymbol{R})$ as a subspace of $C\left(\beta\left(K_{p}\right)\right)\left(=C\left(K_{p}\right)\right)$ and assume that $\|l\|=1$. The Hahn-Banach theorem and the Riesz Representation theorem imply that there exists a $\nu \in \operatorname{rca}\left(\beta\left(K_{p}\right)\right)$ such that $\|\nu\|=1$ and

$$
\langle l, f\rangle=\int_{\beta\left(K_{p}\right)} f(T, \dot{\phi}) d \nu(T, \dot{\phi}), \quad f \in M^{p}(\boldsymbol{R}) .
$$

Suppose that $l$ attains its norm on $g \in S\left(M^{p}(\boldsymbol{R})\right)$, i.e., $\langle l, g\rangle=\|g\|=$ $\|l\|=1$, and let

$$
B=\left\{(T, \dot{\phi}) \in \beta\left(K_{p}\right):|g(T, \dot{\phi})|=1\right\} .
$$

Note that $\nu$ vanishes outside $B$. For each $(T, \phi) \in B$, there exists a net $\left\{\left(T_{\gamma}, \phi_{\gamma}\right)\right\}$ in $K_{p}$ which converges to $(T, \phi)$. Let $\psi^{\prime}=|g|^{p-1} \operatorname{sgn} \bar{g}$. Then $\lim _{\gamma}\left|A\left(T_{\gamma}, g \phi_{\gamma}\right)\right|=1=\lim _{\gamma} A\left(T_{\gamma}, g \gamma\right)$. By the uniform convexity of $L^{q}\left(\left[-T_{r}, T_{r}\right], d x / 2 T_{r}\right)$ (note that each $L^{q}$ has the same modulus of convexity) and Lemma 2.1 , we conclude that $\lim _{\gamma} A\left(T_{\gamma},\left|\psi-\phi_{\gamma}\right|^{q}\right)=0$. This, combined with the Hölder inequality, implies that $\lim _{\gamma} A\left(T_{\gamma}, f \dot{\phi}_{\gamma}\right)=$ $\lim _{\gamma} A\left(T_{\gamma}, f \psi\right)$ for all $f \in M^{p}(\boldsymbol{R})$, and hence $f(T, \phi)=f(T, \psi)$ for all $f \in M^{p}(\boldsymbol{R}),(T, \dot{\rho}) \in B$. Now, for any $f \in M^{p}(\boldsymbol{R})$,

$$
\begin{aligned}
& |\langle l, f\rangle\rangle^{\prime}=\left|\int_{B} f\left(T, \phi^{\prime}\right) d \nu(T, \dot{\phi})\right| \\
& \leqq\|\nu\| \cdot \sup \{|f(T, \dot{\phi})|:(T, \dot{\phi}) \in B\} \\
& =\sup \{|f(T, \psi)|:(T, \dot{\phi}) \in B\} \\
& \leqq \sup \{|f(T, \psi)|: T \geqq 1\} \text {. }
\end{aligned}
$$

If $\tau(f)=\sup \left\{|f(T, \psi)|: T \in \boldsymbol{R}^{+}\right\}, f \in C\left(\beta\left(K_{p}\right)\right), \tau$ is a nonnegative, positive homogeneous subadditive functional. An application of the Hahn-Banach theorem yields a norm preserving extension, $\tilde{\mu} \in$ rea $\left(\beta\left(K_{p}\right)\right)$, of $l$ such that $|\langle\tilde{\mu}, f\rangle| \leqq \tau(f)$ for all $f$ in $C\left(\beta\left(K_{p}\right)\right)$. It follows that $\|\tilde{\mu}\|=1$ and $\tilde{\mu}$ is supported by $\beta[1, \infty) \times\{\psi\}$. By letting $\mu(E)=\tilde{\mu}(E \times\{\psi\})$ for each Borel subset $E$ of $[, \infty)$

$$
\langle l, f\rangle=\int_{1}^{\infty} A(T, f \psi) d u(T) \quad \forall f \in M^{p}(\boldsymbol{R}) .
$$

The fact that $\mu$ is positive follows from $\|\mu\|=1,\|g\|=1$ and $\int_{1}^{\infty} A\left(T,|g|^{p}\right) d \mu(T)=1$.

Let $K$ be a topological space. For each $\mu \in \mathrm{rba}(K), \mu$ can be decomposed as $\mu=\mu_{1}+\mu_{2}$ where $\mu_{1} \in \operatorname{rca}(K)$ and $\mu_{2}$ is purely finitely additive, i.e., if $0 \leqq \nu \leqq\left|\mu_{2}\right|$ and $\nu \in$ rca $(K)$, then $\nu=0$. Note that $\mu_{2}$ vanishes on compact sets of $K$. 
COROLlary 4.4. Let $1<p<\infty$ and let $l$ be a norm attaining functional in $I^{p}(\boldsymbol{R})^{*}$. Then there exists $a \psi \in S\left(I^{q}(\boldsymbol{R})\right)$ and a positive $\mu \in \operatorname{rca}[1, \infty)$ such that $\|\mu\|=\|l\|$ and

$$
\langle l, f\rangle=\int_{1}^{\infty} A(T, f \psi) d \mu(T) \quad \forall f \in I^{p}(\boldsymbol{R}) .
$$

Proof. Let $l \in I^{p}(\boldsymbol{R})^{*}$ with $\|l\|=1$ and let $g \in S\left(I^{p}(\boldsymbol{R})\right)$ such that $\langle l, g\rangle=\|g\|=\|l\|=1$. If $\psi=|g|^{p-1} \operatorname{sgn} \bar{g}$ and $\tilde{l}$ is the norm preserving extension of $l$ on $M^{p}(\boldsymbol{R})$, then by Lemma 4.3, there exists a positive $\tilde{\mu} \in \operatorname{rba}[1, \infty)$ such that $\|\tilde{l}\|=\|\tilde{\mu}\|$,

$$
\langle\tilde{l}, f\rangle=\int_{0}^{\infty} A(T, f \psi) d \tilde{\mu}(T) \quad \forall f \in M^{p}(\boldsymbol{R}) .
$$

Let $\tilde{\mu}=\mu+\mu^{\prime}$ where $\mu \in \operatorname{rca}[1, \infty)$ and $\mu^{\prime}$ is purely finitely additive. Note that $\int_{1}^{\infty} A(T, f \psi) d \mu^{\prime}=0$ for all $f$ in $I^{p}(\boldsymbol{R})$ and we have

$$
\langle l, f\rangle=\int_{1}^{\infty} A(T, f \psi) d \mu(T) \quad \forall f \in I^{p}(\boldsymbol{R}) .
$$

Since $\|\mu\| \leqq 1$ and $\int_{1}^{\infty} A\left(T,|g|^{p}\right) d \mu(T)=\langle l, g\rangle=\|g\|=1$, it follows that the norm of $\mu$ is 1 . This completes the proof.

Since $S\left(I^{p}(\boldsymbol{R})\right.$ ) contains no extreme point (Proposition 3.7), it follows that $I^{p}(\boldsymbol{R})$ is not a dual space. However, the above corollary implies the following more interesting result.

THEOREM 4.5. For $1<p<\infty, I^{p}(\boldsymbol{R})^{* *}=M^{p}(\boldsymbol{R})$.

Proof. Let $\sigma$ be the weak topology on $M^{p}(\boldsymbol{R})$ induced by $I^{p}(\boldsymbol{R})^{*}$. We will show that: (i) For each $f \in M^{p}(\boldsymbol{R}),\|f\|=$ $\sup \left\{\langle l, f\rangle: l \in S\left(I^{p}(\boldsymbol{R})^{*}\right)\right\}$; (ii) $I^{p}(\boldsymbol{R})$ is $\sigma$-dense in $M^{p}(\boldsymbol{R})$; (iii) Every bounded net in $I^{p}(\boldsymbol{R})$ has a $\sigma$-convergent subnet in $M^{p}(\boldsymbol{R})$. It then follows that $I^{p}(\boldsymbol{R})^{* *}=M^{p}(\boldsymbol{R})$.

To prove (i), we let $f \in M^{p}(\boldsymbol{R})$ with $\|f\|=1$. Let $\varepsilon>0$ and suppose that $T_{0}$ satisfies $A\left(T_{0},|f|^{p}\right)>1-\varepsilon$. Let $\psi=|f|^{p-1} \operatorname{sgn} \bar{f}$ and let $\mu=\delta_{T_{0}}$, the point mass measure at $T_{0}$. If $l_{0}$ is the functional defined by $\psi$ and $\mu$ as in Proposition 4.1, then

$$
1-\varepsilon \leqq\left\langle l_{0}, f\right\rangle \leqq \sup \left\{\langle l, f\rangle: l \in S\left(I^{p}(\boldsymbol{R})^{*}\right)\right\} \text {. }
$$

Conversely, if $D$ is the set of norm attaining functionals in $S\left(I^{p}(\boldsymbol{R})^{*}\right)$, then the theorem of Bishop and Phelps [5] implies that $D$ is dense in $S\left(I^{p}(\boldsymbol{R})^{*}\right)$. Corollary 4.4 implies that each $l \in D$ can be represented in terms of $\psi \in S\left(I^{q}(\boldsymbol{R})\right)$ and a positive $\mu \in \mathrm{rca}\left(\boldsymbol{R}^{+}\right)$. Hence 


$$
\langle l, f\rangle=\int_{1}^{\infty} A(T, f \psi) d \mu \leqq \int_{1}^{\infty} A\left(T,|\psi|^{q}\right)^{1 / q} d \mu \leqq 1
$$

By taking the supremum of the left hand side, part (i) follows. To prove (ii), let $f \in M^{p}(\boldsymbol{R})$ be given. For any $l \in S\left(I^{p}(\boldsymbol{R})^{*}\right)$ and for any $\varepsilon>0$, choose $l^{\prime} \in D$ such that $\left\|l-l^{\prime}\right\| \leqq \varepsilon /\|f\|$, where $l^{\prime}$ is represented by $\mu$ and $\psi$ as in Corollary 4.4. There exists a compact set $K$ in $\boldsymbol{R}^{+}$such that $\mu\left(\boldsymbol{R}^{+} \backslash K\right)<\varepsilon /\|f\|$. If $f_{K}=f \cdot \chi_{K \cup(-K)}$, then $f_{K} \in I^{p}(\boldsymbol{R})$ and

$$
\begin{aligned}
\mid\langle l, f\rangle-\left\langle l, f_{K}\right\rangle & \leqq\left|\langle l, f\rangle-\left\langle l^{\prime}, f\right\rangle\right|+\left|\left\langle l^{\prime}, f\right\rangle-\left\langle l^{\prime}, f_{K}\right\rangle\right|+\left|\left\langle l^{\prime}, f_{K}\right\rangle-\left\langle l, f_{K}\right\rangle\right| \\
& \leqq \varepsilon+\int_{R^{+}} A\left(T,\left(f-f_{K}\right) \psi\right) d \mu+\varepsilon \\
& \leqq 3 \varepsilon .
\end{aligned}
$$

To prove (iii), let $\left\{f_{\alpha}\right\}$ be a net in $S\left(I^{p}(\boldsymbol{R})\right)$. For each $n$, the weak compactness of $L^{p}[-n, n]$ and an application of the diagonal method imply that there exists a subnet $\left\{f_{\beta}\right\}$ of $\left\{f_{\alpha}\right\}$ and a locally $L^{p}$ function $f$ such that $f_{\beta} \cdot \chi_{[-n, n]} \stackrel{w^{*}}{\longrightarrow} f \cdot \chi_{[-n, n]}$ for each $n$. Since $A\left(T,\left|f_{\beta}\right|^{p}\right) \leqq 1$, it follows that $A\left(T,|f|^{p}\right) \leqq 1$ and therefore $f \in M^{p}(R)$. The dominated convergence theorem yields that

$$
\lim _{\beta} \int_{1}^{\infty} A\left(T,\left(f_{\beta}-f\right) \psi\right) d \mu=0
$$

for any $\phi \in M^{q}(\boldsymbol{R})$ and $\mu \in \mathrm{rca}\left(\boldsymbol{R}^{+}\right)$. Corollary 4.4 and the density of $D$ in $S\left(I^{p}(\boldsymbol{R})^{*}\right)$ imply that $\left\{f_{\beta}\right\}$ converges to $f$ in the $\sigma$-topology.

Theorem 4.6. Let $1<p<\infty$ and let $l \in I^{p}(\boldsymbol{R})^{*}$. Then there exists a $\vartheta^{\prime} \in S\left(M^{q}(\boldsymbol{R})\right)$ and a positive $\mu \in \mathrm{rca}[1, \infty)$ such that $\|\mu\|=\|l\|$ and

$$
\langle l, f\rangle=\int_{1}^{\infty} A(T, f \psi) d \mu(T) \quad \forall f \in I^{p}(\boldsymbol{R}) .
$$

Proof. Since $M^{p}(\boldsymbol{R})=I^{p}(\boldsymbol{R})^{* *}$, there exists a $g \in S\left(M^{p}(\boldsymbol{R})\right)$ such that $\langle l, g\rangle=\|l\|$. Let $\psi=|g|^{p-1} \operatorname{sgn} \bar{g}$ and let $\tilde{l}$ be the norm preserving extension of $l$ on $M^{p}(\boldsymbol{R})$. By Lemma 4.3, there exists a positive $\tilde{\mu} \in \operatorname{rba}[1, \infty)$ such that $\tilde{l}$ can be represented by $\tilde{\mu}$ and $\psi$. The same argument as in Corollary 4.4 yields

$$
\langle l, f\rangle=\int_{1}^{\infty} A(T, f \psi) d \mu(T) \quad \forall f \in I^{p}(\boldsymbol{R})
$$

where $\mu$ is the countably additive component of $\tilde{\mu}, \mu$ is positive and $\|\mu\|=\|l\|$.

TheOREM 4.7. For $1<p<\infty, M^{p}(\boldsymbol{R})^{*}=I^{p}(\boldsymbol{R})^{*} \oplus I^{p}(\boldsymbol{R})^{\perp}$ and $\left\|l_{1}+l_{2}\right\|=\left\|l_{1}\right\|+\left\|l_{2}\right\|$ for $l_{1} \in I^{p}(\boldsymbol{R})^{*}$ and $l_{2} \in I^{p}(\boldsymbol{R})^{\perp}$. 
Proof. Since $M^{p}(\boldsymbol{R})=I^{p}(\boldsymbol{R})^{* *}$, it follows that $M^{p}(\boldsymbol{R})^{*}=I^{p}(\boldsymbol{R})^{*} \oplus$ $I^{p}(\boldsymbol{R})^{\perp}$. To prove the second assertion, we may assume that $\left\|l_{1}\right\|=$ $\left\|l_{2}\right\|=1$. For $\varepsilon>0$, choose $f_{1} \in I^{p}(\boldsymbol{R}), f_{2} \in M^{p}(\boldsymbol{R})$ such that $\left\langle l_{i}, f_{i}\right\rangle \geqq$ $1-\varepsilon$ and $\left\|f_{i}\right\|=1$. Note that

$$
\varlimsup_{T \rightarrow \infty} A\left(T,\left|f_{2}\right|^{p}\right)=1 .
$$

Without loss of generality, assume that supp $f_{1} \subseteq[-a, a]$ for some $a>0$ such that $\mu(R \backslash(a, \infty))<\varepsilon$ where $\mu$ is the measure in the representation of $l_{1}$ and

$$
A\left(T,\left|f_{1}\right|^{p}\right)<\varepsilon^{p} \quad \text { for all } T>a .
$$

Let $f=f_{1}+f_{2} \cdot \chi_{R \backslash\left[-a, a_{j}\right.}$. Then $\|f\|=\sup _{1 \leqq T} A\left(T,|f|^{p}\right)^{1 / p} \leqq 1+\varepsilon$. The fact that $\left\langle l_{2}, g\right\rangle=0$ for all $g \in M^{p}(\boldsymbol{R})$ with compact support implies that

$$
\begin{aligned}
\left\langle l_{1}+l_{2}, f\right\rangle & \geqq\left(\left\langle l_{1}, f_{1}\right\rangle-\varepsilon\right)+\left\langle l_{2}, f_{2} \cdot \chi_{R /[-a, a]}\right\rangle \\
& =\left\langle l_{1}, f_{1}\right\rangle+\left\langle l_{2}, f_{2}\right\rangle-\varepsilon \\
& \geqq 2-3 \varepsilon .
\end{aligned}
$$

It follows that $\left\|l_{1}+l_{2}\right\| \geqq(2-3 \varepsilon) /(1+\varepsilon)$ and since $\varepsilon$ is arbitrary, $\left\|l_{1}+l_{2}\right\|=2$. This completes the proof.

5. Representation of $\mathscr{C}^{p}(\boldsymbol{R})^{*}$. A finitely additive measure $\mu \in \operatorname{rba}[1, \infty)$ is said to be concentrated at $\infty$ if $\mu(E)=0$ for any measurable subset $E$ contained in a finite interval. It is easy to show that for $1<p<\infty$, if $\psi \in \mathscr{M}^{q}(\boldsymbol{R}), \mu \in \operatorname{rba}[1, \infty)$ and $\mu$ is concentrated at $\infty$, then

$$
\langle l, f\rangle=\int_{1}^{\infty} A(T, f \psi) d \mu(T) \quad \forall f \in \mathscr{M}^{p}(\boldsymbol{R})
$$

defines a functional on $\mathscr{M}^{p}(\boldsymbol{R})$. We will show that every norm attaining functional on $\mathscr{A}^{p}(\boldsymbol{R})$ is of this form.

Recall that $\mathscr{C}^{p}(\boldsymbol{R})$ is isometric isomorphic to $M^{p}(\boldsymbol{R}) / I^{p}(\boldsymbol{R})$ (Proposition 2.4). This implies that $\mathscr{A}^{p}(\boldsymbol{R})^{*}$ is isometric isomorphic to $I^{p}(\boldsymbol{R})^{\perp}$.

LemMA 5.1. Let $1<p<\infty$. Then for each $\bar{f} \in M^{p}(\boldsymbol{R}) / I^{p}(\boldsymbol{R})$, there exists an $f \in M^{p}(\boldsymbol{R})$ such that $\|f\|=\|\bar{f}\|$.

Proof. Theorem 4.7 implies that $I^{p}(\boldsymbol{R})$ is an $M$-ideal [1] in $M^{p}(\boldsymbol{R})$. Hence it is a proximinal subspace of $M^{p}(\boldsymbol{R})$, i.e., for each $f \in M^{p}(\boldsymbol{R})$, there exists a $g \in I^{p}(\boldsymbol{R})$ such that

$$
\|f-g\|=\inf \left\{\|f-h\|: h \in I^{p}(\boldsymbol{R})\right\} .
$$


It follows that each $\bar{f} \in M^{p}(\boldsymbol{R}) / I^{p}(\boldsymbol{R})$ is the image of an $f \in M^{p}(\boldsymbol{R})$ such that $\|f\|=\|\bar{f}\|$.

THEOREM 5.2. Let $1<p<\infty$ and let $l$ be a norm attaining functional on $\mathscr{C}^{p}(\boldsymbol{R})$. Then there exists $a \psi \in \mathscr{C}^{q}(\boldsymbol{R}), 1 / p+1 / q=1$, and a positive $\mu \in \mathrm{rba}[1, \infty)$ which is concentrated at $\infty$ such that

$$
\langle l, f\rangle=\int_{1}^{\infty} A(T, f \psi) d \mu \quad \forall f \in \mathscr{L}^{p}(\boldsymbol{R}) .
$$

Proof. We will assume that $\|l\|=1$. The identification of $\mathscr{C}^{p}(\boldsymbol{R})^{*}$ and $I^{p}(\boldsymbol{R})^{\perp}$, and Lemma 5.1 enable one to assume that $l \in I^{p}(\boldsymbol{R})^{\perp}$ and $l$ attains its norm on a $g \in S\left(M^{p}(\boldsymbol{R})\right)$. Recalling the notation and proof of Lemma 4.3, we claim that for $(T, \phi) \in B$, if $\left\{\left(T_{\gamma}, \dot{\phi}_{\gamma}\right)\right\}$ is a net in $K_{p}$ which converges to $(T, \phi)$, then $\lim _{\gamma} T_{\gamma}=\infty$. This holds, since if not, there is a subnet $\left\{T_{\alpha}\right\}$ such that $\lim _{\alpha} T_{\alpha}=$ $T_{0}<\infty$. If $g_{n}=g \cdot \chi_{R \backslash[-n, n]}$, then $\left\langle l, g_{n}\right\rangle=\langle l, g\rangle=1$ (for $\left.l \in I^{p}(\boldsymbol{R})^{\perp}\right)$. This implies that $\left|g_{n}(T, \dot{\varphi})\right|=1$. But for $n>T_{0}$, there exists an $\alpha_{0}$ such that for $\alpha>\alpha_{0}$,

$$
\left|g_{n}\left(T_{\alpha}, \dot{\phi}_{\alpha}\right)\right| \leqq A\left(T_{\alpha},\left|g_{n}\right|^{p}\right)^{1 / p} \cdot A\left(T_{\alpha},\left|\dot{\phi}_{\alpha}\right|^{q}\right)^{1 / q}=0
$$

Hence $\left|g_{n}(T, \dot{\phi})\right|=0$. This is a contradiction and the claim is proved. It follows that one can show that

$$
|\langle l, f\rangle| \leqq \varlimsup_{T \rightarrow \infty}|f(T, \psi)| \quad \forall f \in M^{p}(\boldsymbol{R})
$$

Moreover, using the proof of Lemma 4.3, one can find a $\mu \in \operatorname{rba}[1, \infty)$ such that

$$
\langle l, f\rangle=\int_{1}^{\infty} A(T, f \psi) d \mu(T) \quad \forall f \in M^{p}(\boldsymbol{R})
$$

with $\mu$ positive and $\|l\|=\|\mu\|$. Inequality (5.1) clearly implies that $\mu$ is concentrated at $\infty$. By considering (5.2) with $f \in \mathscr{M}^{p}(\boldsymbol{R})$, we have is $\in \mathscr{M}^{q}(\boldsymbol{R})$ such that

$$
\langle l, f\rangle=\int_{1}^{\infty} A(T, f \psi) d \mu(T) \quad \forall f \in \mathscr{L}^{p}(\boldsymbol{R}) .
$$

Corollary 5.3. Let $1<p<\infty$ and let $X$ be a closed subspace of $\mathscr{L}^{p}(\boldsymbol{R})$. Then there exists a norm dense subset $D$ in $X^{*}$ such that each $l \in D$ can be represented as in equation (5.2).

Proof. Let $D$ be the set of norm attaining functionals in $X^{*}$. Let $l \in D$. By the Hahn-Banach theorem, $l$ can be extended to a functional $\tilde{l}$ in $\mathscr{C l}^{p}(\boldsymbol{R})^{*}$ with $\|\widetilde{l}\|=\|l\|$ and $\tilde{l}$ also attains its norm. 
The representation of $\tilde{l}$ on $\mathscr{L}^{p}(\boldsymbol{R})$ will give the representation for $l$ on $X$.

We are unable to represent every functional $l$ in $\mathscr{A}^{p}(\boldsymbol{R})^{*}$ as in (5.2). However, if we consider the subspace

$$
\mathscr{M}_{r}^{p}(\boldsymbol{R})=\left\{f \in \mathscr{C}^{p}(\boldsymbol{R}): \lim _{T \rightarrow \pm \infty} \frac{1}{T} \int_{T}^{T+1}|f|^{p}=0\right\},
$$

the space of $\mathscr{M}^{p}$-regular functions, a complete representation can be obtained. The method is due to Cwikel [7, Erratum].

Lemma 5.4. For $1<p<\infty$, let $f \in M_{r}^{p}(\boldsymbol{R}), \phi \in M_{r}^{q}(\boldsymbol{R})$ and suppose that $|S-T|<1$. Then $|A(T, f \dot{\phi})-A(S, T \dot{\phi})| \rightarrow 0$ uniformly as $T \rightarrow \infty$.

Proof. The lemma follows from the following inequality:

$$
\begin{aligned}
\mid A(T, f \dot{\phi}) & -A(S, f \dot{\phi}) \mid \\
& \leqq\left|\frac{1}{2 T} \int_{-T}^{T} f \phi-\frac{1}{2 S} \int_{-T}^{T} f \dot{\phi}\right|+\frac{1}{2 S} \int_{\left[-T T T^{T}[-S S]\right.}|f \phi| \\
& \leqq\left|1-\frac{T}{S}\right| A(T, f \dot{\phi})+\frac{T}{S} \cdot \frac{1}{2 T}\left(\int_{-(T+1)}^{-T}+\int_{T}^{T+1}|f \dot{\phi}|\right)
\end{aligned}
$$

Theorem 5.5. Let $1<p<\infty$ and let $l \in \mathscr{M}_{r}^{p}(\boldsymbol{R})^{*}$. Then there exists a positive $\mu \in \mathrm{rba}[1, \infty)$ which is concentrated at $\infty$ and a two variable Borel measurable function $\psi(T, x)$ such that for each fixed $T, \psi(T, \cdot) \in \mathscr{A}_{r}^{q}(\boldsymbol{R})$ and

$$
\langle l, f\rangle=\int_{1}^{\infty}\left(\frac{1}{2 T} \int_{-T}^{T} f(x) \psi(T, x) d x\right) d \mu(T) \quad \forall f \in \mathscr{A}_{r}^{p}(\boldsymbol{R}) .
$$

Proof. Let $I_{m}=[m, m+1), \quad m \geqq 1$, and partition $I_{m}$ into $2 m+1$ disjoint consecutive subintervals $E_{1, m}, \cdots, E_{2 m+1, m}$. If $E_{n}=$ $\mathrm{U}_{m \geqq n}\left(E_{2 n, m} \cup\left(-E_{2 n, m}\right)\right)$, then $\left\{E_{n}\right\}$ is a disjoint sequence of sets. Applying the notation and proof as in Lemma 4.3 and Theorem 5.2, with $M_{r}^{p}(\boldsymbol{R})$ in place of $M^{p}(\boldsymbol{R})$, we have for each norm attaining functional $l$ on $\mathscr{A}_{r}^{p}(\boldsymbol{R})$, there exists a $g \in S\left(M_{r}^{p}(\boldsymbol{R})\right)$ such that $\langle l, g\rangle=\|l\|$ and

$$
|\langle l, f\rangle| \leqq \varlimsup_{T \rightarrow \infty}|f(T, \phi)|=\varlimsup_{T \rightarrow \infty}|f(T, \phi)| \cdot \chi_{E_{n}} \quad \forall f \in M_{r}^{p}(\boldsymbol{R})
$$

where $\phi=|g|^{p-1} \operatorname{sgn} \bar{g}$ (the last equality follows from Lemma 5.5). Hence we can choose a representation of $l$ with $\phi \in M_{r}^{q}(\boldsymbol{R})$ and a $\nu \in \operatorname{rba}[1, \infty)$ which is supported by $E_{n}$ and concentrated at $\infty$. 
Now, for any $l \in \mathscr{C l}_{r}^{p}(\boldsymbol{R})^{*}$, let $\left\{l_{n}\right\}$ be a sequence of norm attaining functionals which converges to $l$. Suppose that the $l_{n}$ 's are represented by (5.2):

$$
\left\langle l_{n}, f\right\rangle=\int_{1}^{\infty} A\left(T, f \psi_{n}\right) d \nu_{n}(T) \quad \forall f \in M_{r}^{p}(\boldsymbol{R}),
$$

where $\psi_{n} \in M_{r}^{q}(\boldsymbol{R})$ and $\nu_{n}$ is supported by $E_{n}$ and is concentrated at $\infty$. If one defines

$$
\psi(T, x)=\sum_{n=1}^{\infty} \psi_{n}(x) \chi_{E_{n}}(T)
$$

Then it follows that

$$
\left\langle l_{n}, f\right\rangle=\int_{1}^{\infty}\left(\frac{1}{2 T} \int_{-T}^{T} f(x) \psi(T, x) d x\right) d \nu_{n}(T) \quad \forall f \in M_{r}^{p}(\boldsymbol{R}) .
$$

The weak compactness of the unit sphere of $\mathrm{rba}[1, \infty)$ allows one to assume $\mu$ is a $w^{*}$-limit point of $\left\{\nu_{n}\right\}$ and hence

$$
\langle l, f\rangle=\int_{1}^{\infty}\left(\frac{1}{2 T} \int_{-T}^{T} f(x) \psi(T, x) d x\right) d \mu(T) \quad \forall f \in M_{r}^{p}(\boldsymbol{R}) .
$$

It follows immediately that $\mu$ is concentrated at $\infty$ and $\mu$ is positive. By considering $l \in \mathscr{L}_{r}^{p}(\boldsymbol{R})^{*}$, we have $\psi(T, \cdot) \in \mathscr{M}_{r}^{q}(\boldsymbol{R})$

$$
\langle l, f\rangle=\int_{1}^{\infty}\left(\frac{1}{2 T} \int_{-T}^{T} f(x) \psi(T, x)\right) d \mu(T) \quad \forall f \in \mathscr{A}_{r}^{p}(\boldsymbol{R}) .
$$

\section{REFERENCES}

1. E. Alfsen and E. Effros, Structure in real Banach spaces, Ann. of Math., 96 (1972), 98-173.

2. J. Bass, Stationary functions and their applications to the theocy of turbulence, $I, I I$, J. Math. Anal. \& Appl., 47 (1974), 354-399, 458-503.

3. J. Bertrandias, Espaces de Fonctions Bornees et Continues en Moyenne Asymptotique d'order p, Bull. Soc. Math. France, (1966), Memoire 5.

4. A. Besicovitch, Almost Periodic Functions, New York, Dover Publications, 1954.

5. E. Bishop and R. Phelps, A proof that every Banach space is subreflexive, Bull. Amer. Math. Soc., 67 (1961), 97-97.

6. H. Bohr and E. Følner, On some type of functional spaces, Acta Math., 76 (1944), 31-155.

7. M. Cwikel, The dual of weak $L^{p}$, Ann. Inst. Fourier Grenoble, 25 (1975), 81-126. (with Erratum).

8. M. Day, Normed Linear Spaces, Springer.Verlag, New York, 1973.

9. N. Dunford and J. Schwartz, Linear Operator I, Interscience, New York, 1958.

10. K. Lau and J. Lee, On generalized harmonic analysis, Trans. Amer. Math. Soc., 259 (1980), 75-97.

11. J. Marcinkiewicz, Une Remarque sur les Espaces de M. Besicovitch, C. R. Acad. Sci. Paris, 208 (1939), 152-159.

12. P. Masani, Commentary on the memoire on generalized harmonic analysis, [30a], 
Nobert Wiener: Collected Work Vol. II, Edited by Masani, MIT Press, 1979, 333-379. 13. N. Wiener, Generalized harmonic analysis, Acta Math., 55 (1930), 117-258.

14. 1959.

Received April 25, 1979 and in revised form August 28, 1979. Partially supported by the Faculty of Arts and Science research grant of the University of Pittsburgh.

University of Pittsburgh

Pittsburgh, PA 15260 


\section{PACIFIC JOURNAL OF MATHEMATICS}

\section{EDITORS}

DoNALD BABBITT (Managing Editor)

University of Galifornia

Los Angeles, California 90024

HUgo RossI

University of Utah

Salt Lake City, UT 84112

C. C. MOORE AND ANDREW OGG

University of California

Berkeley, CA 94720
J. DugunduI

Department of Mathematics

University of Southern California

Los Angeles, California 90007

R. Finn and J. Milgram

Stanford University

Stanford, California 94305

\section{ASSOCIATE EDITORS}
E. F. BeCKenbaCh
B. H. NeumanN
F. WOLF
K. YoSHIDA

\section{SUPPORTING INSTITUTIONS}

UNIVERSITY OF BRITISH COLUMBIA

CALIFORNIA INSTITUTE OF TECHNOLOGY

UNIVERSITY OF CALIFORNIA

MONTANA STATE UNIVERSITY

UNIVERSITY OF NEVADA, RENO

NEW MEXICO STATE UNIVERSITY

OREGON STATE UNIVERSITY

UNIVERSITY OF OREGON
UNIVERSITY OF SOUTHERN CALIFONIA STANFORD UNIVERSITY UNIVERSITY OF HAWAII UNIVERSITY OF TOKYO UNIVERSITY OF UTAH WASHINGTON STATE UNIVERSITY UNIVERSITY OF WASHINGTON 


\section{Pacific Journal of Mathematics}

\section{Vol. 91, No. $1 \quad$ November, 1980}

Harvey Leslie Abbott, Extremal problems on nonaveraging and nondividing

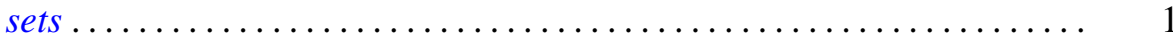

Marine Bruce Abrahamse and Stephen D. Fisher, Mapping intervals to

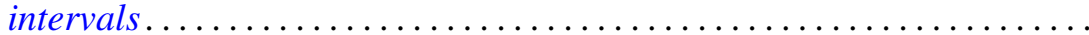

William Wells Adams, The best two-dimensional Diophantine

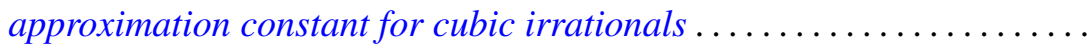

Marilyn Breen, A quantitative version of Krasnosel'skiu 's theorem in

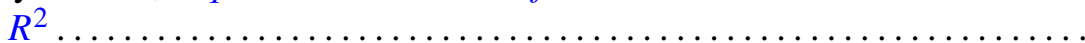

Stephen LaVern Campbell, Linear operators for which $T^{*} T$ and $T T^{*}$

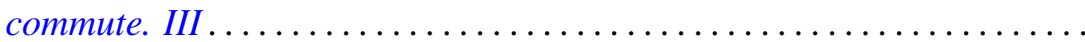

Zvonko Cerin, On cellular decompositions of Hilbert cube manifolds ......

J. R. Choike, Ignacy I. Kotlarski and V. M. Smith, On a characterization

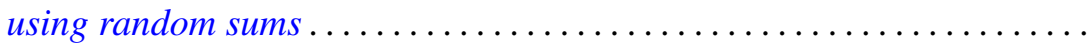

Karl-Theodor Eisele, Direct factorizations of measures .............. 79

Douglas Harris, Every space is a path component space ............. 95

John P. Holmes and Arthur Argyle Sagle, Analytic H-spaces, Campbell-Hausdorff formula, and alternative algebras.............

Richard Howard Hudson and Kenneth S. Williams, Some new residuacity criteria ..........................................

V. Karunakaran and Michael Robert Ziegler, The radius of starlikeness for a class of regular functions defined by an integral ....

Ka-Sing Lau, On the Banach spaces of functions with bounded upper

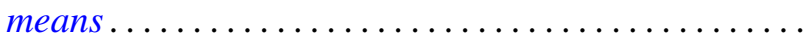

Daniel Paul Maki, On determining regular behavior from the recurrence formula for orthogonal polynomials................

Stephen Joseph McAdam, Asymptotic prime divisors and going down...

Douglas Edward Miller, Borel selectors for separated quotients ..

Kent Morrison, The scheme of finite-dimensional representations of an algebra

Donald P. Story, A characterization of the local Radon-Nikodým property by tensor products

Arne Stray, Two applications of the Schur-Nevanlinna algorithm ...

N. B. Tinberg, The Levi decomposition of a split $(B, N)$-pair ...

Charles Irvin Vinsonhaler and William Jennings Wickless, A theorem on quasi-pure-projective torsion free abelian groups of finite rank... 